\title{
Discontinuous decompaction of a falling sandpile
}

\author{
J. Duran, ${ }^{1}$ T. Mazozi, ${ }^{1}$ S. Luding, ${ }^{1,2}$ E. Clément,${ }^{1}$ and J. Rajchenbach ${ }^{1}$ \\ ${ }^{1}$ Laboratoire d'Accoustique et d'Optique de la Matière Condensée, URA 800, \\ Université Pierre et Marie Curie, 4 place Jussieu, 75005 Paris, France \\ ${ }^{2}$ Theoretische Polymerphysik, Rheinstrasse 12, D-79104 Freiburg, Germany
}

(Received 28 July 1995)

\begin{abstract}
We investigate experimentally and via computer simulations the fall of a two dimensional granular material in a rectangular container with friction only at the lateral walls. We study the decompaction modes of the granular assembly, which is a basic question relevant to the general dynamics of a noncohesive powder. We observe during the fall the possible occurrence of successive cracks splitting the initial pile into smaller blocks as time passes. These cracks preferentially occur in the lower part of the array, resulting in an ascending decompaction wave in the bulk. We show experimentally how this effect is related to the surface roughness of the boundaries. A heuristic continuum theory is proposed that rationalizes the experimental results. Furthermore, event driven simulations, including particle rotations and friction, parallel nicely the experimental observations. In the simulations, we find strong pressure fluctuations at the boundaries correlated to the occurrence of arches.
\end{abstract}

PACS number(s): 46.10.+z, 83.70.Fn, 47.20.-k

\section{INTRODUCTION}

Among other open questions concerning the physics of granular materials (see $[1,2]$ and references therein), the problem of guided flows in tubes, pipes, or chutes is of crucial importance. The great majority of industrial processes dealing with granulates is influenced by several basic phenomena. Effects like recurrent clogging [3] as a sort of "traffic jam" problem [4], density waves [5-8], and size segregation [9-11] are frequently observed during granulate processing. Related to industrial challenges and to fundamental questions, experimental efforts have been attempted to clarify the intriguing problem of the behavior of sand flowing along inclined chutes either in three dimensional (3D) situations [12] or in 2D model granular materials [13] $[14,15]$. However, due to the difficulty of dealing with particle-wall interactions and also due to the subsequent formation of stress chains in the granulate, the system is not yet completely understood and undoubtedly requires further experimental, theoretical, and simulation work. Some publications deal with granular flows and particularly with propagating density waves within falling powders. Density waves have been found in simulations to occur in a steady state regime $[5,8]$ or in experiments, related to gas-particle interactions (pneumatic effects) [16]. In the spirit of current efforts dealing with model granular materials $[17,11,18,19,15]$ we tackle the problem of a $2 \mathrm{D}$ pile made up of rather large beads, in order to minimize the influence of the surrounding gas. Since $2 \mathrm{D}$ model systems greatly reduce the geometrical complexity existing in commonly used granular materials, it is likely that some difference shows up when comparing model media results with more general sand problems. However, the increasing amount of experimental data shows that many basic features of the physics of granulates are maintained in model situations. This is due to the common nature of microscopic granular interactions (frictional and dissi- pative collisions). In contrast to the more complex real situation, such an approach has two main advantages: first, it allows a direct observation of the behavior of the falling column of particles, and second, it permits a tractable computer simulation to be made which, in turn, can be confronted to experimental observations on quantitative grounds.

Recent observations of approximately V-shaped microcracks (see Fig. 1) in vertically vibrated sandpiles [17]

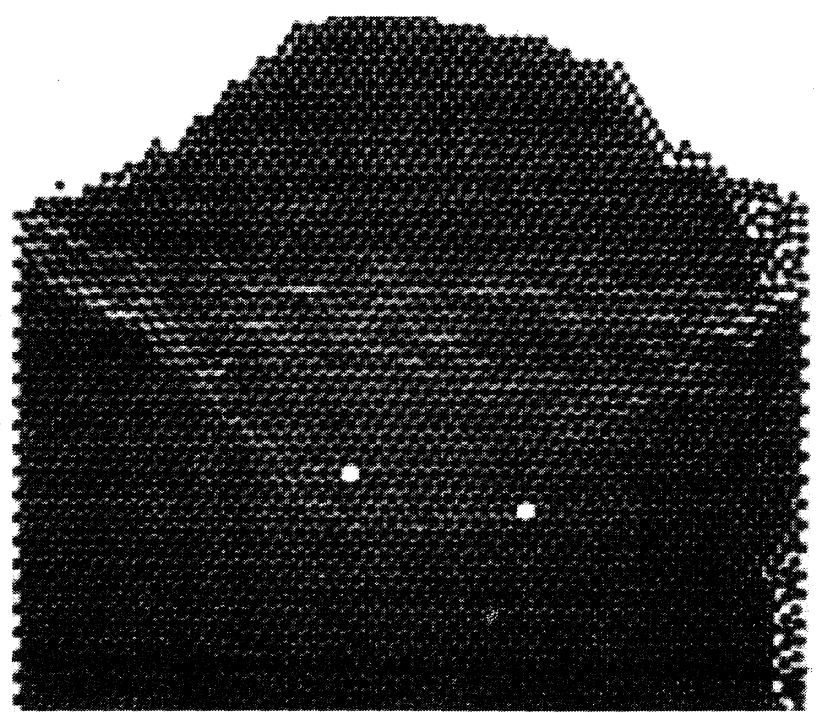

FIG. 1. Experimental observation of successive microfractures which occur in a vertically vibrated (at $15 \mathrm{~Hz}) 2 \mathrm{D}$ pile in a box. The micro-cracks occur repeatedly and erratically with an approximative $V$ shape during a few milliseconds at every period of excitation when the compact pile moves upwards. This photograph has been obtained by using a series of stroboscopic flash light synchronized with the vibration period at a given phase. 
lead us to the problem of gravity driven vertical motion of sandpiles in $2 \mathrm{D}$ containers. In a continuously vibrated container with rough walls, the cracks appear periodically at different positions in the pile and exist only during a short interval of time (e.g., for a few milliseconds at a vibration frequency of $15 \mathrm{~Hz}$ ). Therefore, we designed an experiment which allows a much longer observation of the dynamics of these microcracks. Hence, we study the fall of a $2 \mathrm{D}$ granular material inside a rectangular container with frictional lateral walls. We examine the modes of decompaction, which is a basic problem related to the general dynamics of a noncohesive powder. This paper reports a series of experiments, paralleled by computer simulations based on an event-driven algorithm, including rough and rotating particles. We discuss the basic features of both, simulation and experiments, using an extension of our previously developed continuum model [17].

\section{EXPERIMENTS AND SIMULATIONS}

\section{A. Experimental details}

For the experiments, we use a set of monodisperse oxidized aluminum beads of diameter $d=1.5 \mathrm{~mm}$, which we have already considered in previous works as a convenient model granular material $[10,17,18]$. These metallic beads are initially prepared in an ordered triangular network inside a vertical $2 \mathrm{D}$ cell made up of two glass windows for visualization and two lateral vertical walls of plexiglass. The width of the cell is typically $3.6 \mathrm{~cm}$ and the heights of the arrays range from 0.15 to $19 \mathrm{~cm}$, while the gap between the glass windows is marginally greater than the diameter of the beads. This setup minimizes friction with the front and back windows while maintaining strong friction between the pile and the lateral boundaries and thus mimics a convenient $2 \mathrm{D}$ granular object. For a schematic picture of the experimental setup see Fig. 2.

The cell containing the pile is initially closed at its lower outlet by a vertical and $1 \mathrm{~mm}$ thin aluminum blade which can be moved downward at an acceleration of approximately $3 g$, i.e., larger than the gravitational acceleration $g$. This aluminum blade acts as a piston which is abruptly pulled down via a strong vertical spring. The whole setup is carefully aligned in order to make sure that the piston does not touch the cell when moving. Since the downwards air drag, obtained when the piston is lowered, might induce artificial effects at the beginning of the falling process, we checked for this by testing that a single bead or a single layer of beads falls according to the acceleration of gravity $g$, so that we will neglect the influence of the surrounding air in the following.

The recording setup consists of a charge coupled device (CCD) camera interfaced to an image processing device. The definition of the origin of time, i.e., the time when the fall starts, is appreciated via an additional setup which uses a He-Ne laser, the beam of which is cut off when the blade starts moving downwards.

Our observation and recording of a large number of experiments in different containers leads to the conclusion

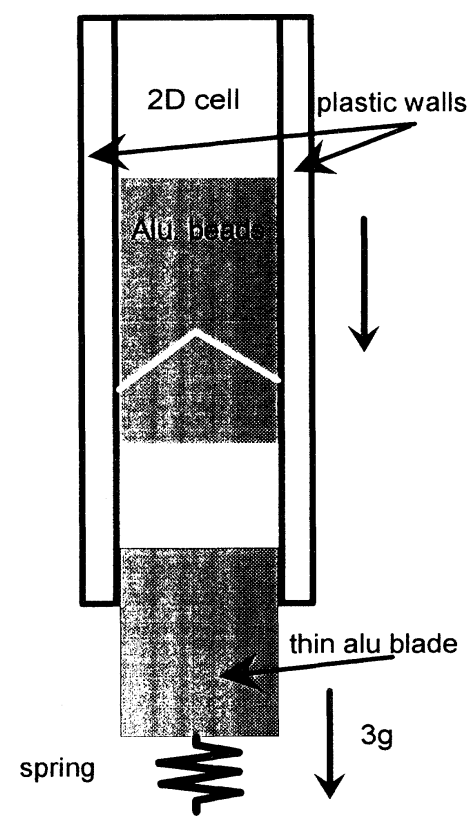

FIG. 2. Schematic drawing of the experimental setup.

that during the fall discontinuous decompaction may occur via cracks. In Fig. 3 we present snapshots of a typical experiment (a) complemented by the result of numerical simulations (b). For details on the simulations and the parameters used therein see the following subsection. The development of the cracks starts in the lower part of the pile and ascends progressively inside the bulk in both experiment and simulation.

Examining our experimental data we get in summary two important features which will be considered in more detail in Sec. III.

(i) Almost perfectly machined, i.e., optically polished, laterals walls are unlikely to induce cracks during the fall, so that the pile often remains compact. On the contrary, rather poorly polished walls unavoidably introduce cracks which display the following characteristics.

(ii) If a crack occurs in the lower portion of the pile, it quite generally increases in size as the fall proceeds. In contrast, if a crack happens to occur in the upper part, it tends to close and disappear during the fall.

Concerning point (i), we checked that both differently machined walls (i.e., optically polished or not) did exhibit approximately the same bead-wall coefficient of friction $\mu_{w}$. The consequences of this fact will be discussed in Sec. III.

In order to complement and extend our experimental observations, we set up a computer simulation which takes into account both the bead-bead as well as the bead-wall friction.

\section{B. Simulations}

Our computer simulation model is based on the following considerations: Since a rough surface implies both rotation and energy loss, it is important to allow rotation 
and to account for the friction of the particles. In the simulations, we describe the roughness of surfaces and the connected energy dissipation, using the coefficient of friction $\mu$, and the maximum tangential restitution $\beta_{0}$, as already described in Refs. [20-22]. Other mechanisms of energy loss might be a permanent deformation of a particle during contact, or the transfer of kinetic energy to thermal energy. We account for these effects, introducing the coefficient of normal restitution, $\epsilon$.

\section{Interaction model}

First, we give a brief description of the collision model we use. For a more detailed description see Refs. [20-22]. Consider two particles with diameter $d_{1}$ and $d_{2}$ and masses $m_{1}$ and $m_{2}$. The normal unit vector for their contact is $\vec{n}=\frac{\vec{r}_{1}-\vec{r}_{2}}{\left|\vec{r}_{1}-\vec{r}_{2}\right|}$, where $\vec{r}_{i}$ is the vector to the center of particle $i(i=1,2)$. For the interaction of particle 1 with a wall, we set $m_{2}=\infty, d_{2}=0$ and $\vec{n}$ is in this case the unit vector from the contact point with the wall to the center of the particle. The relative velocity of the contact points is $\vec{v}_{c}=\vec{v}_{1}-\vec{v}_{2}-\left(\frac{d_{1}}{2} \vec{\omega}_{1}+\frac{d_{2}}{2} \vec{\omega}_{2}\right) \times \vec{n}$, with $\vec{v}_{i}$ and $\vec{\omega}_{i}$ being the linear and angular velocities of particle $i$ just before collision. From the momentum conservation laws for linear and angular momentum we get

$$
\vec{v}_{1}^{\prime}=\vec{v}_{1}+\Delta \vec{P} / m_{1}
$$

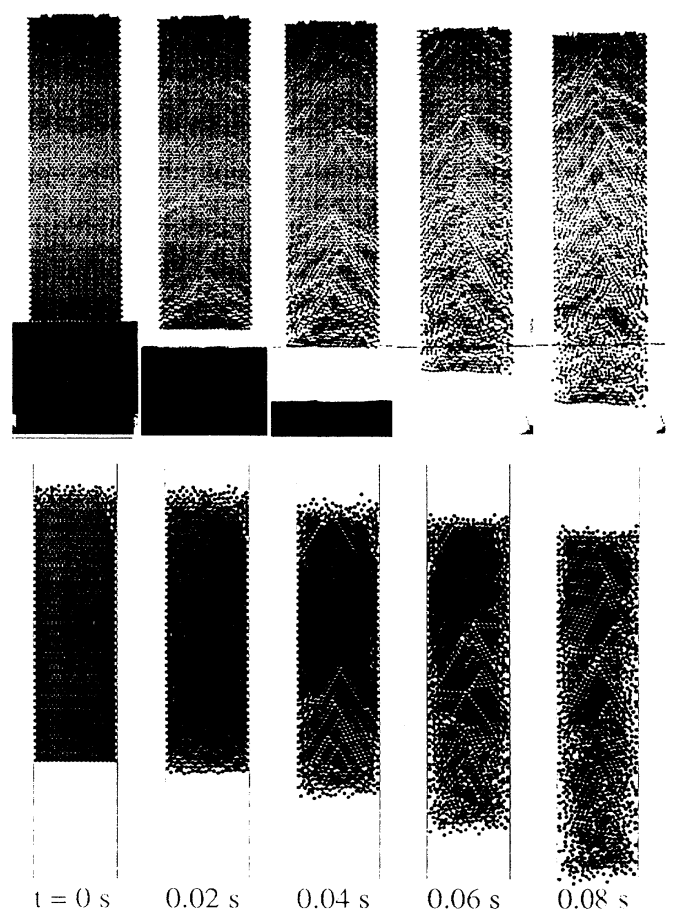

FIG. 3. (a) Experiment: Successive snapshots (aperture time $1 / 2000 \mathrm{~s}$ ) taken at $0.02 \mathrm{~s}$ intervals after the piston has been removed. We use $L=24 d$ and 103 layers so that $S_{0} \approx 3.7$. (b) Simulation: In a container of $L=20.2 d$ and with 80 layers so that $S_{0} \approx 3.5$ we use $\epsilon=0.99, \epsilon_{w}=0.98, \mu$ $=0.5, \mu_{w}=1.0, \beta_{0}=\beta_{0 w}=0.2$, and $\bar{v}(t=0) \approx 0.05 \mathrm{~m} / \mathrm{s}$.

$$
\begin{gathered}
\vec{\omega}_{1}^{\prime}=\vec{\omega}_{1}-\frac{d_{1}}{\left(2 I_{1}\right)} \vec{n} \times \Delta \vec{P} \\
\vec{v}_{2}^{\prime}=\vec{v}_{2}-\Delta \vec{P} / m_{2}, \\
\vec{\omega}_{2}^{\prime}=\vec{\omega}_{2}-\frac{d_{2}}{\left(2 I_{2}\right)} \vec{n} \times \Delta \vec{P}
\end{gathered}
$$

where $\vec{v}_{i}^{\prime}$ and $\vec{\omega}_{i}^{\prime}$ are the unknown velocities of particle $i$ after the collision. $I_{i}$ is the moment of inertia about the center of particle $i$ and $\Delta \vec{P}$ is the change of linear momentum of particle 1 and is a function of $\epsilon, \mu$, and $\beta_{0}$ :

$$
\Delta \vec{P}=-m_{12}(1+\epsilon) \vec{v}_{c}^{(n)}-\frac{2}{7} m_{12}(1+\beta) \vec{v}_{c}^{(t)},
$$

with the reduced mass $m_{12}=m_{1} m_{2} /\left(m_{1}+m_{2}\right)$. (n) and $(t)$ indicate the normal and the tangential component of $\vec{v}_{c}$, respectively, and the factor $2 / 7$ in the tangential part of Eq. (2) stems from the fact that we use solid spheres. $\epsilon$ is the (constant) coefficient of normal restitution and, in parallel we also have $\beta$, the coefficient of tangential restitution. We simplify the two particle contacts in the sense that we allow exclusively Coulomb-type interactions, i.e., $\Delta P^{(t)}$ is limited by $\mu_{w} \Delta P^{(n)}$, or broken contacts with the maximum tangential restitution $\beta_{0}$ due to the elasticity of the material. For a detailed discussion of the above equations see Ref. [22].

\section{Simulation method}

For the simulation we use an event driven (ED) method such that the particles undergo an undisturbed motion in the gravitational field until an event occurs. An event is either the collision of two particles or the collision of one particle with a wall. From the velocities just before contact, we compute the particles velocities after a contact following Eqs. (1). In the ED method, the time for which two particles are in contact is implicitly zero, the consequence being that exclusively pair contacts occur. Thus ED algorithms run into problems when the time between events, $t_{e v}$, gets too small. In systems with strong dissipation $t_{e v}$ may even vanish and the so-called "inelastic collapse" may occur [23-26]. In Ref. [27] an ED algorithm was introduced which updates only those particles which were involved in the previous collision. For this a double buffering data structure is implemented together with several other techniques in order to optimize the speed of calculation. A more detailed description of the original algorithm is given in Ref. [27]. Like in Refs. $[22,28]$ we implement the algorithm of Ref. [27] with some changes and extensions. Despite the gravitational acceleration, all contact times of particles with each other or with the lateral walls can be calculated analytically. If, for example, the coefficient of normal restitution depends on the partner of the colliding particle, we use $\epsilon$ or $\epsilon_{w}$ for particle-particle or particle-wall collisions, respectively. For more details on the collision model see Refs. [20-22] and Sec. II B 1. 


\section{Initial conditions}

We are interested in the situation when a rather compact array of particles begins to fall and progressively decompacts. We use a box of width $L$ and initially arrange $N$ particles with diameter $d$ on a triangular lattice with lattice constant $s=1.01 d$. Each particle gets a random velocity, uniformly distributed in the range $-v_{0} \leq v_{i}(0) \leq v_{0}$ in both horizontal and vertical direction. This rather regular system is now allowed to reach a steady state, i.e., we start the simulation at $t=-t_{r}$, using $\epsilon=\epsilon_{w}=1$ and $\mu=\mu_{w}=0$. A typical average velocity in our simulations is $\bar{v}=\sqrt{\left\langle v^{2}\right\rangle}=0.05 \mathrm{~m} / \mathrm{s}$ for $t=0$. Due to the rather low kinetic energy, the array of particles is still arranged on a triangular lattice, except for a few layers at the top which are fluidized [see Fig. 3(b)]. In a typical simulation, we use $L=20.2 d$ and $N=1562$, so that the array consists of about 80 layers. At time $t=0$ we remove the bottom, switch on dissipation and friction and let the array decompact. We used different initial conditions, keeping all other parameters fixed and found strong fluctuations in position and shape of the cracks. However, the cracks are a reproducible effect, almost independent of the initial conditions. When decreasing the tangential friction $\mu_{w}$, the cracks vanish since the pile falls with only weak perturbations from the lateral walls.

\section{Numerical results \\ 1. Density}

In Fig. 4 we plot the density, i.e., the packing fraction (defined as the ratio of density and close packed density), along the vertical axis, obtained from experiments (a) and from simulations (b) for the snapshots shown in Fig. 3. The comparison between both calculated and experimental profiles can be considered as fair provided two unavoidable artifacts are taken into consideration.

(i) The experimentally obtained packing fraction suffers from imperfection due to inhomogeneities in the lighting of the screen placed behind the experimental setup and which tends to darken somewhat the upper and the lower parts of the snapshots, see Fig. 3(a).

(ii) The ED algorithm implies some "artificial" thermal agitation which leads to the fluidization of a few layers in the top of the pile. The finite kinetic energy in the system thus leads to a smooth decrease of density at the top. In ED simulations the pile is not static, i.e., the particles are always fluctuating around their positions in the triangular lattice. Note that this erratic motion is the only source of fluctuations in the numerical procedure.

Since the comparison between experiments and simulations seems satisfactory, we investigate via simulations the problem of stress in the pile $[20,29]$ and particularly the problem of forces the pile exerts on the lateral walls during the fall.

\section{Pressure at the lateral walls}

From simulations, we get the pressure on the side walls by integrating the normal component of the momentum
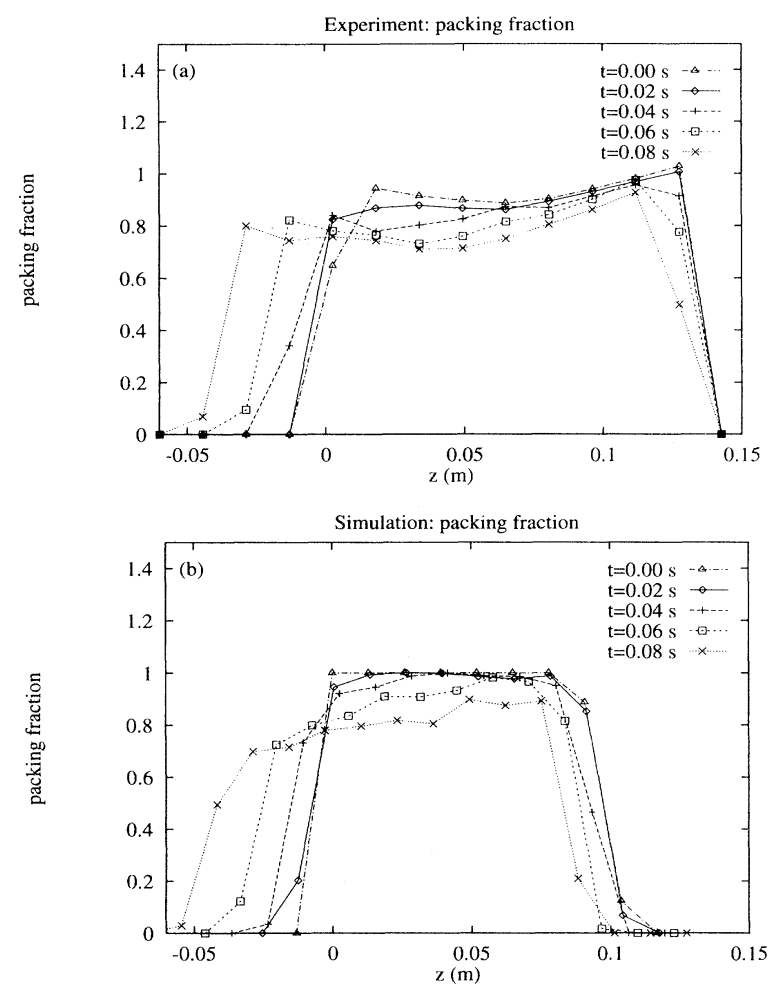

FIG. 4. (a) Experiment: Packing fraction versus height from a typical experiment. (b) Simulation: Packing fraction versus the vertical coordinate from the simulations presented in Fig. 3(b).

change, Eq. (2), of those particles colliding with the wall within the time $t-\Delta t$ and $t$. In Fig. 5 we choose $\Delta t=$ $0.01 \mathrm{~s}$ and plot the pressure as of function of height so that each data point represents the pressure on a part of the wall about six layers high. For $t=0.02 \mathrm{~s}$ (diamonds) we still have a rather small pressure, whereas for $t=0.04$ $\mathrm{s}$ (crosses) the pressure increases over almost one order of magnitude in the lower part of the pile. As can be intuitively imagined, the increase in pressure is strongly

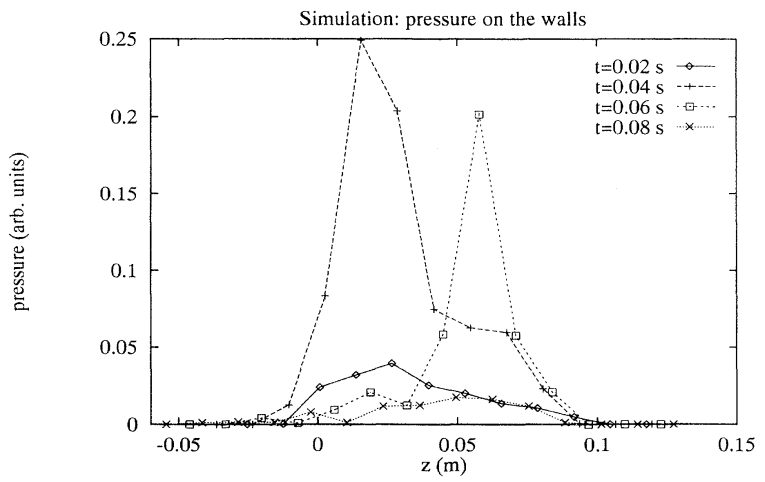

FIG. 5. Plot of the pressure on the side walls as a function of height, obtained from the numerical simulations of Fig. 3(b). The integration interval is $\Delta t=0.01 \mathrm{~s}$ and each data point corresponds to a wall segment about six layers high. 
correlated to the occurrence of cracks. During the fall of the array arches, vaults or, in other words, contact chains may build up corresponding in our simulations, to a large number of collisions per unit time and thus to a great amount of momentum change. These arches hold the particles above, at least for a short time, and thus allow a crack to open below. As can be seen in Fig. 5 , these arches happen to disappear, after a short while, thereby allowing the pressure to relax and the falling to proceed further. At time $t=0.06 \mathrm{~s}$ (squares) we observe cracks also in the upper part of the pile, again connected to strong pressure. For even longer times, $t=0.08 \mathrm{~s}$ ( $\times$ symbols) the particles are already too dilute near the walls, such that contact chains are not longer probable.

\section{THEORY AND RESULTS}

In view of the experimental and simulational results obtained under different conditions, and also in the spirit of our preceding work on vibrational progressive decompaction [17], we set up a model which allows us to understand several of the observed features as well as suggesting a further test proving the pertinence of the model which aims at describing the influence of the lateral beadwall friction, $\mu_{w}$, and of the shape factor $K$ of the $2 \mathrm{D}$ pile.

We recall here our basic model [17] for a two dimensional array of granular material of width $L$ and height $h_{0}$. The height $h$ is measured starting from the lowest row of the rectangular pile. This two dimensional granulate is allowed to fall between two infinite vertical walls which interact with the granular material via a Coulomb friction. Using arguments previously put forward by Janssen [30] and revisited later by Lord Rayleigh [31], we get an equation for the vertical and downward force $P(h)$ acting on a slice $d m$ of material at height $h$ :

$$
P(h)=\frac{\rho_{0} g L}{2 K \mu_{w}}\left\{1-\exp \left[\frac{2 K \mu_{w}}{L}\left(h-h_{0}\right)\right]\right\},
$$

where $\rho_{0}$ is the linear density of the granulate, $\mu_{w}$ is the coefficient of friction for particle-wall contacts, and $K$ is the dimensionless parameter which characterizes the conversion of vertical into horizontal stress due to the imbricated nature of the granulate, for details see Ref. [17].

Equation (3) simply states the fact that the stress in the material depends linearly on the depth in the upper part of the pile, i.e., $P\left(h_{0}-h\right) \approx \rho_{0} g h$ for small $h$, while it tends to saturate to $P_{\max }=\rho_{0} g L /\left(2 K \mu_{w}\right)$ in the lower part of the granulate. This equation can also be interpreted as describing an arching effect which tends to reduce exponentially the downward propagation of a vertical stress with a characteristic length $\zeta=L /\left(2 K \mu_{w}\right)$ which we call the vault range. As in Sec. II C 2, vaults or arches are chains of contacts which possibly span the space between the lateral boundaries. In the following, we will show that this peculiar stress distribution [see Eq. (3)], which relies on a basic and rather simplistic description of the sandpile, implies a number of consequences concerning the experiment of a falling pile.

\section{A. How does a compact pile fall?}

During its fall, each slice $d m$ of the compact rectangular sandpile is submitted to the conjugate action of both the downward force $g d m$ corresponding to its weight and to a vertical, upward frictional force at the boundaries, $-d F_{\text {frict }}$. The acceleration $\gamma(h)$ acting upon this slice of material can be written in terms of a reduced acceleration $\Gamma(h)$ :

$$
\gamma(h)=g \Gamma(h)=g-\frac{\partial F_{\text {frict }}}{\partial m} .
$$

The second term in Eq. (4) can be found in the derivation of Eq. (3), (see Ref. [17]):

$$
\frac{\partial F_{\text {frict }}}{\partial m}=\frac{P(h)}{\rho_{0} \zeta}=g\left[1-\exp \left(\frac{h-h_{0}}{\zeta}\right)\right] .
$$

It is noteworthy that we consider here the simplest version of the classic Coulomb definition of the static friction coefficient. Strictly speaking, the above equation (5) is true at the very moment of the beginning of the downward motion, i.e., in the static case. For the sake of simplicity, we will consider in the following that the dynamic coefficient of friction will not depend on velocity and remains identical to the static one. Inserting Eq. (5) into Eq. (4) we find that the slice of material at vertical coordinate $h$ is submitted to a reduced acceleration:

$$
\Gamma(h)=\exp \left(\frac{h-h_{0}}{\zeta}\right), \quad h \in\left[0, h_{0}\right]
$$

We first address the basic question to know whether this model implies that the pile would remain compact during the fall or, in contrast, would break into pieces during the downward motion.

Equation (6) shows that the reduced acceleration is increasing with height $h$ so that the lower part of the pile is less accelerated than the upper part. This results in a tendency of the pile to remain compact unless any parameter or condition changes during the fall. Such changes may be fluctuations in the particle-wall coefficient of friction, in the piling geometry, or in the surface of the lateral walls. Supposing that the pile is compact and homogeneous, its overall reduced acceleration $\Gamma_{0}$ will be determined by the lowest slice of the pile, so that we deduce from Eq. (6):

$$
\Gamma_{0}=\exp \left(-\frac{h_{0}}{\zeta}\right)=\exp \left(-S_{0} K \mu_{w}\right),
$$

where the aspect ratio of the pile is $S_{0}=h_{0} / L$.

In order to test this prediction, we performed several experiments, using different $S_{0}$ values, and we measured the position of the uppermost layer of the pile, i.e., $h_{0}(t)$, as a function of time. These experimental results are gathered in Fig. 6 (squares) and compared to the results of our theoretical model (solid lines) using only a single adjustable parameter $K \mu_{w}$. This parameter has been measured repeatedly under different experimental conditions in our preceding work [17], where we used the same 


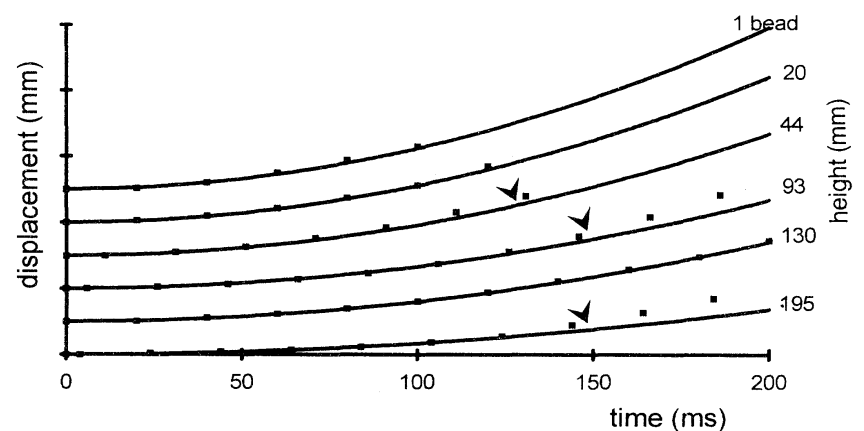

FIG. 6. Plot of the successive positions of the top of the falling piles as function of time. The solid lines represent a fit of the continuum model with $K \mu_{w}=0.12$ to the experimental measurements (squares). The arrows indicate the time when some cracks appear in the bulk. Note that the $130 \mathrm{~mm}$ high pile fell down without showing any visible crack.

aluminum beads and the same material (plexiglass) for the lateral walls. In Ref. [17] the $K \mu_{w}$ values range from 0.11 to 0.29 and were reported to depend on the specific properties of the particles (e.g., wear of the bead surfaces) and the walls. We find that the whole set of data in Fig. 6, obtained in the same container with different heights $h_{0}$ of the granulate, can be fitted with the value $K \mu_{w}=0.12$, according to Eq.(7). As can be observed in Fig. 6, the agreement is satisfactory, at least during the time the pile has stayed compact during the fall. As soon as a crack occurs in the array, we observe, as expected, that the top layer of the pile accelerates more strongly, in agreement with the model, which states that the acceleration increases when the height of the pile decreases. The occurrence of visible cracks is indicated by arrows in Fig. 6 , which also mark the departure from the theoretical model.

As a proof and using nearly perfectly machined lateral walls, we observe that the whole pile falls at a reduced acceleration without showing any cracks, i.e., it stays compact; see the $130 \mathrm{~mm}$ pile in Fig. 6. In order to examine this effect, and as mentioned above (Sec. II A), we use a set of differently prepared lateral walls. Both optically polished and slightly roughened surfaces lead to the same coefficient of friction, $\mu_{w}=0.6$. For measuring $\mu_{w}$, we glued three of our aluminum beads on a disk and get the coefficient of friction by tilting a plate of the wall material, until the disk on top starts to slide. We infer $\mu_{w}$ from the angle of this event. The optically polished walls often do not lead to cracks during the fall. In contrast, poorly polished lateral walls almost always induce cracks starting from the bottom of the pile and ascending in the pile as it proceeds to fall. The loss of optical quality of the surface is connected to heterogeneities of at least $10^{-6} \mathrm{~m}$ size. From this result we infer that fluctuations on the surfaces of the lateral walls induce the cracks. Homogeneous Coulomb friction alone seems to be insufficient to cause the cracks. An additional experiment with a thin strip engraved in the lateral wall leads to cracks starting from this point and thus proves unambiguously that discontinuities at the wall are at the origin of the cracks.

In this section we have shown that the piles behave according to the predictions of the continuous model, as long as no cracks occur. In the following section we will discuss the stability and the position of cracks in the pile.

\section{B. Where do the cracks appear in the falling pile?}

Now, we assume that a crack occurs accidentally in the pile during the downward motion. This may be due to some heterogeneity in the lateral wall friction, or spontaneously created by shocks and collisions. In any case, we realize that such cracks split the pile into two unconnected parts. As a consequence, Eq. (7) may not hold true any more. Now we raise the question of the stability of such an accidental crack. In order to investigate the stability of such a crack and to stay within the limits of our continuum model, we imagine that a horizontal crack occurs during the fall at a vertical coordinate $h_{d}$ in the rectangular array. Considering that this accidental crack lasts long enough to allow the system to reset its internal distribution of stress, we get from Eq. (6) the reduced accelerations of the two resulting rectangular subpiles $A$ (for above) and $B$ (for below):

$$
\begin{aligned}
& \Gamma_{A}=\exp \left(-\frac{h_{0}-h_{d}}{\zeta}\right) \\
& \Gamma_{B}=\exp \left(-\frac{h_{d}}{\zeta}\right)
\end{aligned}
$$

which holds true when $h_{d}$ lies in the interval $\left[0, h_{0}\right]$. It turns out immediately that the condition for getting a crack which will increase in size during the fall, reads as $\Gamma_{B}>\Gamma_{A}$, otherwise the crack would be unstable and would tend to reduce as the time passes. This leads to the elementary condition: $h_{d}<h_{0} / 2$, for the stability of a crack occurring in the pile. Thus our model predicts that stable cracks occur only in the lower half of the rectangular array.

This peculiar feature can be noticed both on experimental observations and on the computer simulation results; see Fig. 3. The cracks start from the bottom of the pile and occur at larger heights only for long times. This concerns also the stability of the cracks occurring in the upper part of the $2 \mathrm{D}$ pile during the fall. As stated in our model, these cracks would tend to reduce as the time passes. In Fig. 3(b) at $t=0.04 \mathrm{~s}$ we observe at the top two small cracks, which are vanished again at $t$ $=0.06 \mathrm{~s}$. The same feature can be also be observed in experimentally obtained snaphots.

Cracks opening in the lower part of the pile do increase in size during the fall, thereby directly confirming our friction-based model. Two subpiles will separate faster if the crack occurs lower in the initial pile. This provides a simple explanation to the experimental fact that a tube filled with sand, and being turned upside down, will display a progressive decompaction of the pile starting from the lowest part. The upper part of the pile stays compact while the sand falls down like rain in the lower part.

Now we derive a general conservation law which links the reduced accelerations $\Gamma_{i}$ of all subpiles due to dis- 
continuous decompaction. From Eq. (8) we first observe that $\Gamma_{A} \Gamma_{B}=\exp \left(-\frac{h_{0}}{\zeta}\right)=\Gamma_{0}$, which can be easily iterated all along the decompaction process. Due to mass conservation which, in this problem, reads as $\sum_{i} h_{i}=h_{0}$ the conservation law for the reduced accelerations of all the subpiles of the pile can be written as

$$
\prod_{i} \Gamma_{i}=\Gamma_{0}=\exp \left(-\frac{h_{0}}{\zeta}\right),
$$

which stands for a rather unusual conservation law which, as far as we know, has no equivalent in other fields of physics.

Thus, the present model implies that the cracks will split the pile below the midheight and, preferentially, begin from the lowest rows of the pile. This prediction is confirmed by both experiments and simulations.

\section{SUMMARY AND DISCUSSION}

We presented experiments and simulations on a $2 \mathrm{D}$ granular system, falling inside a vertical and rectangular container. We observe no steady regime for the falling dynamics: the granular assembly decompacts progressively and accelerates down. Looking in detail at the geometrical patterns formed by the grains, we observe either a discontinuous decompaction or a continuous one. The discontinuous decompaction is the result of cracks breaking the array into pieces from the bottom to the top. Both experiments and simulations verify two basic predictions deduced from a continuum theory based on a dynamic extension of Janssen's vault model [30]. First, as long as no cracks occur, the theory predicts the acceleration of the top of the pile as a function of the aspect ratio: for increasing height, the acceleration of the top decreases. Second, if some crack occurs, it is stable only in the lower half of the pile and both blocks, hence detached, will fall with a larger acceleration and will separate further. Experiments show that cracks rarely show up whenever the lateral walls are optically polished; cracks always appear for a surface roughness larger than typically $10^{-6} \mathrm{~m}$. On the other hand, in simulations, the surface is perfectly flat. Nevertheless, cracks are always observed for coefficients of friction close to the experimental value. As mentioned in Sec.II B 2, the ED algorithm requires the use of a nonzero thermal agitation in order to provide dynamical interactions by collisions. This eventually introduces fluctuations in the system that may cause cracks, even when the coefficient of friction is constant and the wall is flat. In order to test whether cracks are primarily induced by external fluctuations such as heterogeneities at the lateral walls, we suggest a comparison with molecular dynamics calculations, which do not explicitly require an internal noise. Moreover, the simulations have shown (Sec. II C 2) another important feature: strong pressure fluctuations are connected to the occurrence of arches and cracks and thus are propagating upwards.

It is noteworthy to point out that the discontinuous decompaction, presented here, is by no means in contradiction with the recently described progressive decompaction [17]. As a matter of fact, the progressive decompaction model refers to observations made over large periods of time. In other words, progressive decompaction stands for an observation, over a large number of successive perturbations, and of the superposition of a large number of discontinuous decompaction processes.

As expected, we observe that the process of falling down is equivalent to the upward motion of a pile in a continuously vibrated box as illustrated in Fig. 1. Comparing Figs. 1 and 3 we see that the cracks, originated by the side walls, are oriented to oppose to the motion of the particles relatively to the lateral walls. The cracks are approximately following the geometry of the triangular network such that they occur mainly at angles of $30^{\circ}$ relative to the walls. Note that we also observe a weak proportion of cracks with other angles, see Fig. 3 (b). Thinking in terms of arches or vaults leaning on the lateral walls, we note that the inverse V-shape of the contact chains is consistent with the natural shape of stable arches in a triangular network. This is reminiscent of the concept of "free fall arches" [32], which sustain a plug of granulate above freely falling particles. Along this line, we may tentatively extrapolate our results to $3 \mathrm{D}$ situations by conjecturing that a similar discontinuous decompaction process might occur via the successive formation and destruction of arches. As far as we know, the existence of cracks in monodisperse $3 \mathrm{D}$ as well as in polydisperse systems has not been reported so far.

\section{ACKNOWLEDGMENTS}

We thank A. Blumen and H. J. Herrmann for helpful discussions. S. L. gratefully acknowledges the support of the European Community (Human Capital and Mobility) and of the PROCOPE/APAPE scientific collaboration program. The group is part of the French Groupement de Recherche sur la Matière Hétérogène et Complexe of the CNRS and of a CEE network HCM.
[1] H. M. Jaeger and S. Nagel, Science 255, 1523 (1992).

[2] H. M. Jaeger, J. B. Knight, C. h. Liu, and S. R. Nagel, MRS Bull. 5, 25 (1994).

[3] T. Pöschel, J. Phys. (France) I 4, 499 (1994).

[4] K. Nagel and H. J. Hermann, Physica A 190, 254 (1993).

[5] G. Peng and H. J. Hermann, Phys. Rev. E 49, 1796 (1994).

[6] G. W. Baxter, R. Behringer, T. Fagert, and G. Jonhson,
Phys. Rev. Lett. 62, 2825 (1989).

[7] G. W. Baxter, R. Leone, R. P. Behringer, Europhys. Lett. 21, 569 (1993).

[8] J. Lee and M. Leibig, J. Phys. (France) I 4, 507 (1994).

[9] A. Rosato, K. J. Strandburg, F. Prinz, and R. H. Swendsen, Phys. Rev. Lett. 58, 1038 (1987).

[10] J. Duran, J. Rajchenbach, and E. Clément, Phys. Rev. Lett. 70, 2431 (1993). 
[11] J. B. Knight, H. M. Jaeger, and S. R. Nagel, Phys. Rev. Lett. 70, 3728 (1993).

[12] S. B. Savage, J. Phys. Mech. 92, 53 (1979).

[13] T. G. Drake, J. Geophys. Res. 95, 8681 (1990).

[14] F. X. Riguidel, M. Ammi, D. Bideau, A. Hansen, and J. C. Messager, in Powders \& Grains 93, edited by C. Thornton and A. A. Balkema (Publisher, Rotterdam, 1993).

[15] F. Cantelaube, Y. Limon Duparcmeur, D. Bideau, and G.H.Ristow, J. Phys. (France) I 5, 581 (1995).

[16] T. Raafat, J. P. Hulin, H. J. Herrmann (unpublished).

[17] J. Duran, T. Mazozi, E. Clément, and J. Rajchenbach, Phys. Rev. E 50, 3092 (1994).

[18] E. Clément, J. Duran, and J. Rajchenbach, Phys. Rev. Lett. 69, 1189 (1992).

[19] S. Warr, G. T. H. Jacques, and J. M. Huntley, Powder Tech. 81, 41 (1994).

[20] O. R. Walton and R. L. Braun, J. Rheol. 30, 949 (1986).

[21] S. F. Foerster, M. Y. Louge, H. Chang, and K. Allia,
Phys. Fluids 6, 1108 (1994).

[22] S. Luding, Phys. Rev. E 52, 4442 (1995).

[23] S. Luding et al., Phys. Rev. E 49, 1634 (1994).

[24] B. Bernu and R. Mazighi, J. Phys. A 23, 5745 (1990)

[25] S. McNamara and W. R. Young, Phys. Fluids A 4, 496 (1992).

[26] S. McNamara and W. R. Young, Phys. Fluids A 5, 34 (1993).

[27] B. D. Lubachevsky, J. Comput. Phys. 94, 255 (1991).

[28] S. Luding, H. J. Herrmann, and A. Blumen, Phys. Rev. E 50, 3100 (1994).

[29] J. P. Bouchaud, in Non Linearity and Breakdown in Soft Condensed Matter, edited by K. K. Bardhan, B. K. Chakrabarti, and A. Hansen (Springer-Verlag, Berlin, 1994), Vol. 437, pp. 47-53.

[30] H. A. Janssen, Z. Vereins Deutsch Ing. 39, 1045 (1895).

[31] O. L. Rayleigh, Philos. Mag. 61, 129 (1906).

[32] R. L. Brown and J. C. Richards in Principle of Powder Mechanics (Pergamon Press, Oxford, 1970). 


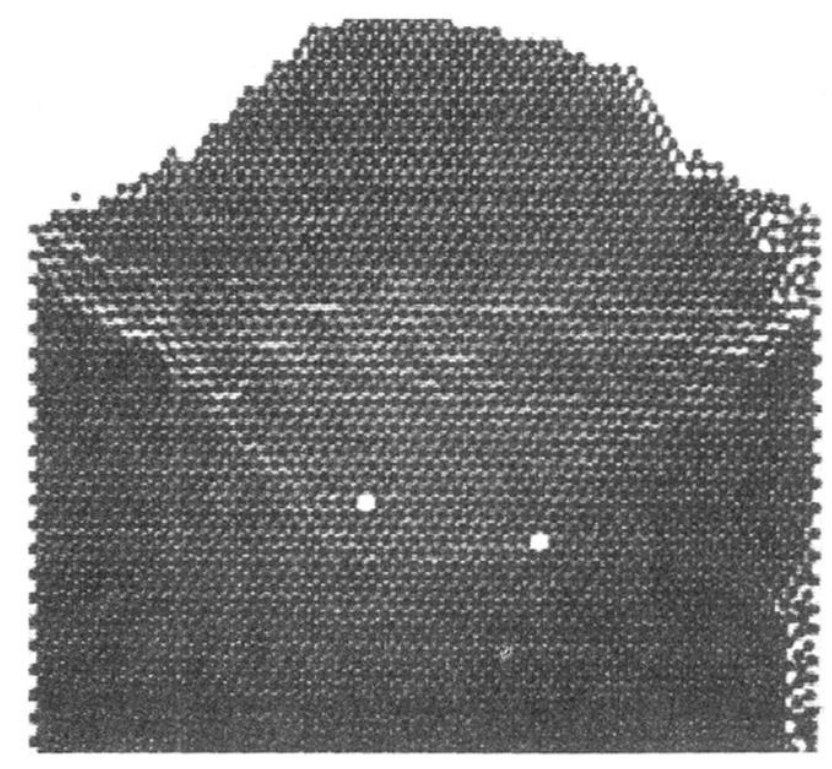

FIG. 1. Experimental observation of successive microfractures which occur in a vertically vibrated (at $15 \mathrm{~Hz}$ ) 2D pile in a box. The micro-cracks occur repeatedly and erratically with an approximative $\mathrm{V}$ shape during a few milliseconds at every period of excitation when the compact pile moves upwards. This photograph has been obtained by using a series of stroboscopic flash light synchronized with the vibration period at a given phase. 


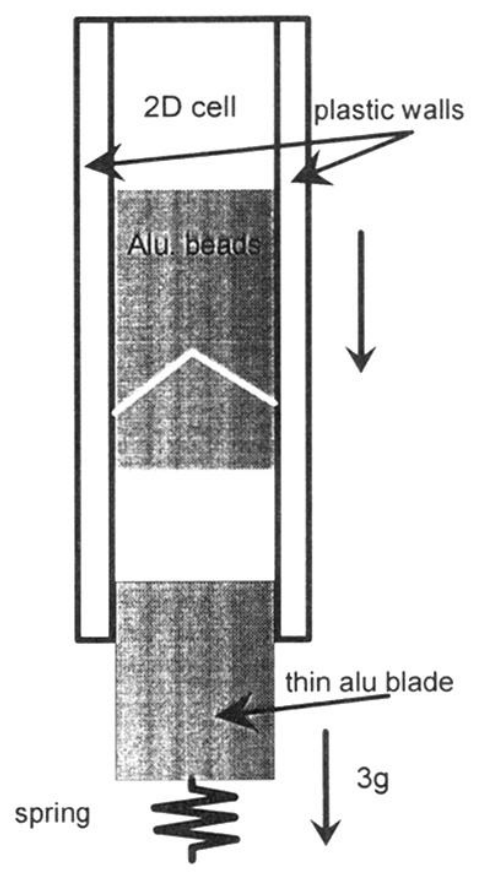

FIG. 2. Schematic drawing of the experimental setup. 


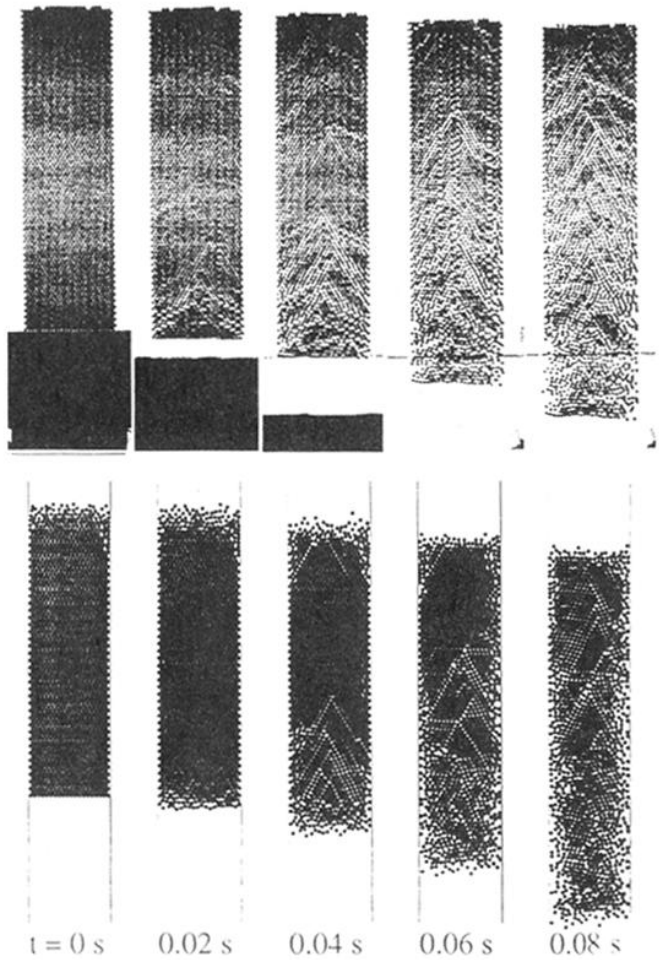

(a)

(b)

FIG. 3. (a) Experiment: Successive snapshots (aperture time $1 / 2000 \mathrm{~s}$ ) taken at $0.02 \mathrm{~s}$ intervals after the piston has been removed. We use $L=24 d$ and 103 layers so that $S_{0} \approx 3.7$. (b) Simulation: In a container of $L=20.2 d$ and with 80 layers so that $S_{0} \approx 3.5$ we use $\epsilon=0.99, \epsilon_{w}=0.98, \mu$ $=0.5, \mu_{w}=1.0, \beta_{0}=\beta_{0 w}=0.2$, and $\bar{v}(t=0) \approx 0.05 \mathrm{~m} / \mathrm{s}$. 